\title{
Catalogue of the primatological collection of the Torino University
}

\author{
Mara Calvini ${ }^{1}$, Maria Stella Siori ${ }^{2}$, Spartaco Gippoliti ${ }^{3}$, Marco Pavia ${ }^{4 *}$
}

\begin{abstract}
The revised catalogue of primatological material stored in the Museo Regionale di Scienze Naturali of Torino and in the Dipartimento di Scienze della Vita e Biologia dei Sistemi of the Università degli Studi di Torino and belonging to the historical material of the Torino University is introduced. The material, 494 specimens belonging to 399 individuals of 122 taxa, is of particular importance since specimens were mainly obtained during the $19^{\text {th }}$ Century and the beginning of the $20^{\text {th }}$ Century. A relevant part of the collection was created by the collaborators of the Museum, among which it is worth to mention F. De Filippi, A. Borelli and E. Festa, while other material came from purchases and donations from private people or the Royal Zoological Garden of Torino. Great part of the specimens is stuffed but also the osteological materials are of particular importance, as many of them derived from the specimens before being prepared and consisting of skulls or more or less complete skeletons. After this revision, the Lectotype and Paralectotypes of Alouatta palliata aequatorialis have been selected, and the type-specimen of the brunnea variety of Cebus albifrons cuscinus has been recognized. In addition, some specimens of particular historical-scientific importance have also been identified and here presented for the first time.
\end{abstract} Torino.

Key words: mammalogy, collection, catalogue, primates,

Riassunto - Catalogo delle Collezioni di Primati dell'Università di Torino.

Viene presentato il catalogo della collezione di Primati presenti al Museo Regionale di Scienze Naturali di Torino e al Dipartimento di Scienze della Vita e Biologia dei Sistemi dell'Università degli Studi di Torino provenienti dalle colle-

${ }^{1}$ Via D. Alighieri 426, 18038 Sanremo (IM), Italia

Email: mara.calvini@gmail.com

2 Dipartimento di Scienze della Vita e Biologia dei Sistemi, Via Accademia Albertina 13, 10123 Torino, Italia

Email: mariastella.siori@unito.it

${ }^{3}$ IUCN/SSC Primate Specialist Group, Viale Liegi 48,

00198 Roma, Italia

${ }^{4}$ Museo di Geologia e Paleontologia, Dipartimento di Scienze della Terra, Via Valperga Caluso 35, 10125 Torino, Italia

* Corresponding author: marco.pavia@unito.it

(C) 2016 Mara Calvini, Maria Stella Siori, Spartaco Gippoliti, Marco Pavia

Received: 14 March 2016

Accepted for publication: 27 July 2016 zioni storiche dell'Università di Torino. Il materiale, costituito da 494 campioni appartenenti a 399 individui di 122 taxa, risulta particolarmente importante da un punto di vista sia storico che scientifico, in quanto comprende esemplari raccolti tra il Secolo XIX e il Secolo XX in molte aree geografiche. Una considerevole parte dei materiali proviene dalle raccolte degli zoologi del Museo di Torino F. De Filippi, A. Borelli e E. Festa, mentre molti reperti provengono da acquisti o donazioni e dal Giardino Zoologico Reale di Torino. Gli esemplari presenti sono principalmente tassidermizzati, anche se non va trascurata la parte osteologica costituita da crani e scheletri montati, in alcuni casi appartenenti allo stesso esemplare di cui è conservata la pelle tassidermizzata. Per tutti gli esemplari è stata fatta una verifica dei cataloghi manoscritti e dei cartellini originali controllando le determinazioni e aggiornando la nomenclatura. Gli esemplari sono stati catalogati con una nuova numerazione composta dall'acronimo MZUT (Museo di Zoologia dell'Università degli Studi di Torino) seguito dalla lettera $\mathrm{T}$ (Teriologia) e da un numero progressivo univoco. I dati presenti, insieme a tutte le numerazioni usate in precedenza, sono stati inseriti in un database di nuova creazione unico per tutte le collezioni MZUT e ogni esemplare è stato munito di un nuovo cartellino. Particolare attenzione è stata rivolta al riconoscimento di diversi campioni, come reperti osteologici ottenuti prima della tassidermizzazione, derivanti da un singolo esemplare e che sono stati riuniti sotto lo stesso numero di catalogo. Questa revisione ha permesso di designare il Lectotipo e i Paralectotipi di Alouatta palliata aequatorialis e l'esemplare-tipo della varietà brunnea di Cebus albifrons cuscinus. Vengono inoltre presentati alcuni esemplari di particolare interesse storico-scientifico, tra cui il primo esemplare noto per un museo europeo di Sapajus flavius e uno scheletro completo di Gorilla gorilla maschio arrivato a Torino nel 1862 e presentato al pubblico da F. De Filippi nel corso della sua conferenza del 1864 "L'uomo e le scimie". Torino.

Parole chiave: teriologia, collezioni, catalogo, primati,

\section{INTRODUCTION}

The primatological collections of the Museo di Zoologia of the Torino University (MZUT) started to grown at the beginning of the $19^{\text {th }}$ Century with the first donations, including those of L. Rolando (1773-1831), the famous anatomist. A great impulse to the primatological collection of Torino has been under the direction of F.A. Bonelli (1784-1830) who obtained some specimens from the Museum de Histoire Naturelle in Paris and from internationally known dealers (Leadbeater, Frank, Bullock). 
The most relevant specimens of the collections were added in the second half of the $19^{\text {th }}$ Century after various scientific expeditions, among which it is worth to mention the material collected by Caffer (voyage of the Regina, 1839-1840) and by De Filippi during the world travel of the "H.M.S. Magenta" (1865-1868), and the specimens provided by Ghiliani, Borelli and Festa after their expeditions in South America. Some specimens also originated from other collectors not directly related to the Torino Museum like Carcano, Crivelli, D'Olivieri, Bovero (South America); Bertoldo, Michela, Bonarelli (South-East Asia); Gariazzo and Neri (Central Africa); Testa Fochi and Muzioli (North-East Africa).

Festa visited Panama and Ecuador in a four-year travel from 1895 to 1898 (Festa, 1909). During this expedition a huge amount of zoological specimens of various animal classes have been collected, some of them were later described as new species (e.g. Salvadori \& Festa, 1899). Festa also published one paper on the primates collected during his expedition (Festa, 1903) in which he also described the new taxon Alouatta aequatorialis, recently considered as still valid (Bigatti et al., 2011), but currently considered as a subspecies of $A$. palliata (Mittermeier et al., 2013).

The primatological specimens collected by Borelli in Argentina, Paraguay, Brazil and Bolivia between 18931896 remained undocumented as those obtained from most other collectors. The collections of the MZUT were also increased with specimens from the Royal Zoological Gardens of Torino and Stupinigi (Maschetti et al., 1988) or from private donors, including famous traveling menageries (Bidel, Tournaire etc.) and animal dealers (such as Molinar).

Other specimens, with also some osteological remains, were added at the beginning of the $20^{\text {th }}$ Century, mainly after the scientific expedition of the Duca degli Abruzzi in Uganda and the purchases of material from Ethiopia, Katanga and Kenya by the taxidermist Bajnotti. After the First World War and the death of L. Camerano (1856-1917) the primatological collections, as the other zoological ones, were object of minor interest, as the zoological studies no longer involved taxonomical aspects and the analysis of the collections. Biographic information on some of the major collectors and donors can be obtained from Andreone et al. (2007).

In 1936, when the Museo di Zoologia and Anatomia Comparata (MACUT) were moved from Palazzo Carignano to the Ospedale San Giovanni Antica Sede, the primate collections, as well as the other numerous twentieth-century zoological collections (around 700 exhibited specimens for the primates only), ended up scattered among different museums and university institutes. This is in fact the same period when the stuffed material were assigned to the Istituto di Zoologia, whereas the osteological material and anatomy preparations were kept at the Istituto di Anatomia Comparata (Ardito \& Bigatti, 2006). After the institution of the Museo Regionale di Scienze Naturali (MRSN) in 1978, with an agreement between Regione Piemonte and the Torino University, a large part of the primatological collection converged to the MRSN. The material was firstly revi- sed by Crovella et al. (1990) but, notwithstanding the good will of the individuals, it is still waiting for a suitable arrangement, due to lack of funds and dedicated people. More recently, the primatological collections have been the subject of scientific papers on their history and some relevant topics (Crovella et al., 1990; Crovella \& Passerin D'Entreves, 1990; Ardito \& Bigatti, 2006; Bigatti et al., 2011; Lastella et al., 2012; Vuch et al., 2013; Giovanardi, 2014), as also were described in popular publications (e.g. Visalberghi \& Valente, 1997).

Here we present the results of the reordering of the primatological collection of the Museo di Zoologia (MZUT) and Museo di Anatomia Comparata (MACUT) of the Torino University.

Further enquiries about this collection and about its specimens should be addressed to the curators of the Zoological Section of the MRSN (zoologia.mrsn@regione.piemonte.it).

\section{MATERIALS AND METHODS}

This catalogue largely follows the structure proposed by Gavetti \& Andreone (1993). The most relevant variation from the already published catalogues of the MRSN material (Gavetti \& Andreone, 1993; Andreone et al., 2007) is that all the University zoological collections now stored at the MRSN have been catalogued with the acronym MZUT, regardless of the former $\mathrm{Mu}-$ seum, both MZUT and MACUT. The acronym MZUT is followed by $\mathrm{T}$ (Theriology) for all the mammal material and by a progressive unique number. The numbers were assigned ex-novo, as many different numberings were assigned to that material during the time. All the published numbers and the number reported in the manuscript catalogue (Catalogo Generale) were reported and, in particular, the former MACUT numbers were reported with the acronym. In the cases were we recognized the presence of different specimens belonging to the same individuals, we put them together under the same MZUT catalogue number. Tags with the new number and labels with number and species name were tied to each specimen.

We critically revised all the data also using the manuscript catalogues and the labels, we also verified most taxonomical determinations and updated the nomenclature. All the data were inputted in the database for all the mammals of the MZUT specifically elaborated for the MRSN by one of the authors (MP) and new labels were prepared for all the specimens. In addition we try to recognize all the specimens belonging to the same individual, in particular the osteological material derived from the specimens before being stuffed (e.g. skin and skeleton), that were labeled under the same catalogue number.

The structure of the catalogue is as follow. The first column of each specimen reports the acronym (MZUT $=$ Museo di Zoologia dell'Università di Torino, $\mathrm{T}=\mathrm{Te}$ riologia) followed by the catalogue number. The second column reports the numbers formerly referred to the specimens; in particular, the numbers are from the old MZUT and MACUT manuscript Catalogues, and 
the various attempts of cataloguing the Primate collections. The third column reports the geographic information, from the state, provinces, municipalities, and toponyms; in addition, when available, the reference date, the collector(s), the donor(s), and the age and sex are given. In absence of any geographical information, we indicate "w.d." (without data). In the fourth column, we indicate the preserved specimens. The specimens are preserved as stuffed, mounted (Mount) or study skin (Skin), or complete or partial skulls and/ or skeletons.

We did not taken into consideration in our work the liquid-preserved anatomical parts (e.g., organs) and the Homo remains. The specimens that were indeterminate in the catalogues and on the labels were left as they were or, whenever possible, attributed to the infraorder.

The nomenclature here adopted follows Groves (2005) and secondary Mittermeier et al. (2013).

The comments within the square brackets are Authors' original notes.

\section{RESULTS}

The collection of MZUT actually comprises 494 specimens belonging to 399 individuals of 122 taxa. In particular, they are 184 stuffed specimens and 183 skulls and 127 more or less complete skeletons.

The values of the specimens can also be seen under a double point of view: expositive and scientific. Within the rich primatological collection housed in Torino some stuffed monkeys (e.g. Pan, Papio, Colobus and Microcebus) may play a considerable expositive role as also a few big skeletons, such as those of Gorilla and Papio. As an example, some mounted lemurs are now seen in the old-fashioned exhibit devoted to Madagascar as a part of the "historical museum of zoology" hall.

It is also worth to mention the Type-series of Alouatta palliata aequatorialis described by Festa (1903) and recently revised by Bigatti et al. (2011), among which we selected here the Lectotype MZUT T 33 (Fig. 1a-b) and the Paralectotypes (MZUT T 31, MZUT T 32, MZUT T 34) (Fig. 1d-f).

During the present work, the type-specimen of $\mathrm{Ce}$ bus albifrons cuscinus var. brunnea Festa, 1903 was also identified (MZUT T 57, Fig. 2). This name escaped attention by primate taxonomists as Festa put it only in the final table of his paper (Festa, 1903: p. 8), but we recognized the importance of this specimen, on the light of future taxonomic revision of the Genus.

Another interesting finding of the present work regards the skin of a Sapajus flavius Schreber, 1774 donated in 1911 (MZUT T 76, Fig. 3). Actually this species has been revalidated and specimens finally examined only a decade ago (De Oliveira \& Langguth, 2006) and thus the MZUT specimen has a particular historical significance as the first museum specimen so far recorded outside Brazil. Noteworthy is also the identification of a specimen (MZUT T 81) of Pithecia pissinatti (Fig. 4), a recently described species by Marsh (2014).
In the original catalogues and labels, the mounted specimen MZUT T 93 (Fig. 5) has been referred to Cercopithecus mitis boutourlinii Giglioli, 1887. This specimen is dated 1885 , i.e. two years before the official description of that species. The detailed analysis of the specimen, also suggested by the fact that Giglioli did not mention the MZUT specimen in his papers (1887, 1888), revealed that MZUT T 93 has to be referred to Semnopithecus vetulus (Erxleben, 1777), a Colobine endemic to Sry Lanka, slightly similar to Cercopithecus mitis boutourlinii.

It is also worth mentioning the complete skeleton of an adult male of Gorilla gorilla (MZUT T 323, Fig. 6), for which it was possible to indirect dating its arrival in Torino in 1862 on the basis of the data reported in the oldest manuscript catalogue of the collection (Catalogo Antico). In fact, this catalogue is strictly in chronological order and the Gorilla gorilla skeleton is between two specimens of which the year 1862 is indicated as the data of acquisition. In addition, during his conference "L'uomo e le scimie" held in Torino in 1864, F. De Filippi presented to the public a complete skeleton of an adult individual, which is most likely the specimen MZUT T 323.

The scientific importance of the Primatological collection of MZUT is also testified by the fact that part of the skeletal material is still kept in the Dipartimento di Scienze della Vita e Biologia dei Sistemi (former Istituto di Zoologia) of the Torino University and used for anatomical and paleontological studies (Mottura \& Ardito, 1992; Gentili et al., 1998; Mottura \& Gentili, 2006; Lastella et al., 2012; Vuch et al., 2013; Giovanardi, 2014). There is in the last two decades a revived interest in taxonomy, as it represent the basis for biodiversity conservation. Hopefully some of the specimens historically collected in South America may furnish additional information for groups in need of revisions, e.g. Cebus and Sapajus (Rylands et al., 2005). In fact, the historical significance of the primatological collection, especially those concerning South America, is relevant and should be better appreciated by visitors and researchers (Gippoliti, 2009).

We hope that the publication of this catalogue will generate a great interest on one of the most important Italian primatological collection, which has been neglected for too many years.

\section{Aknowledgements}

The authors wish to thank to E. De Biaggi, former director of the MRSN, and E. Gavetti, curator in chief of the Zoological section of the MRSN, for the opportunity to study the material housed in the Museo Regionale di Scienze Naturali di Torino. E. Gavetti and L. Ghirardi are also thanked for their invaluable help during the various phases of the work. We also would like to thank P. Passerin D'Entreves for the information about the Gorilla gorilla specimen. We would like to thank S. Boccardi, M. Favelli, M. Garzena, G. Tessa, for their help during the work. M. Calvini has been founded as a contractor of MRSN on this specific topic. 


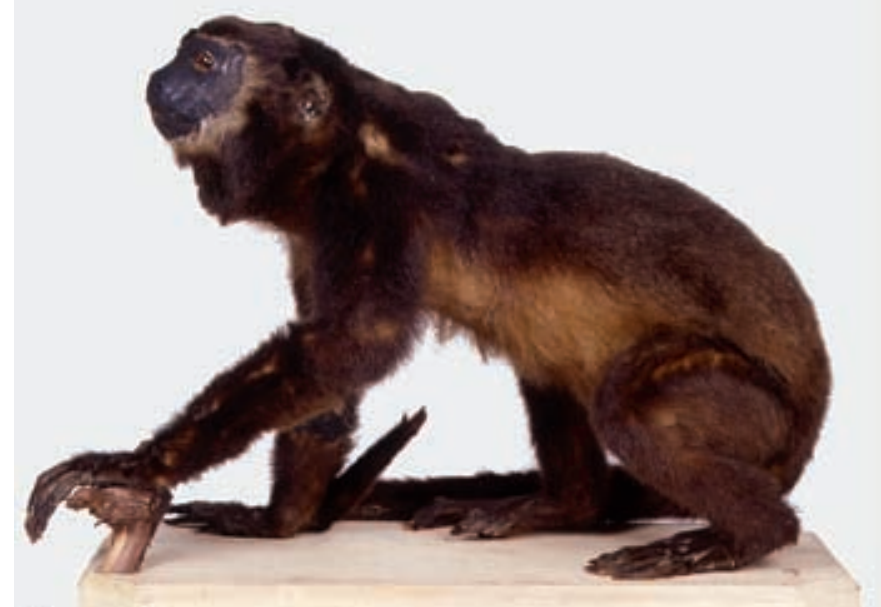

a
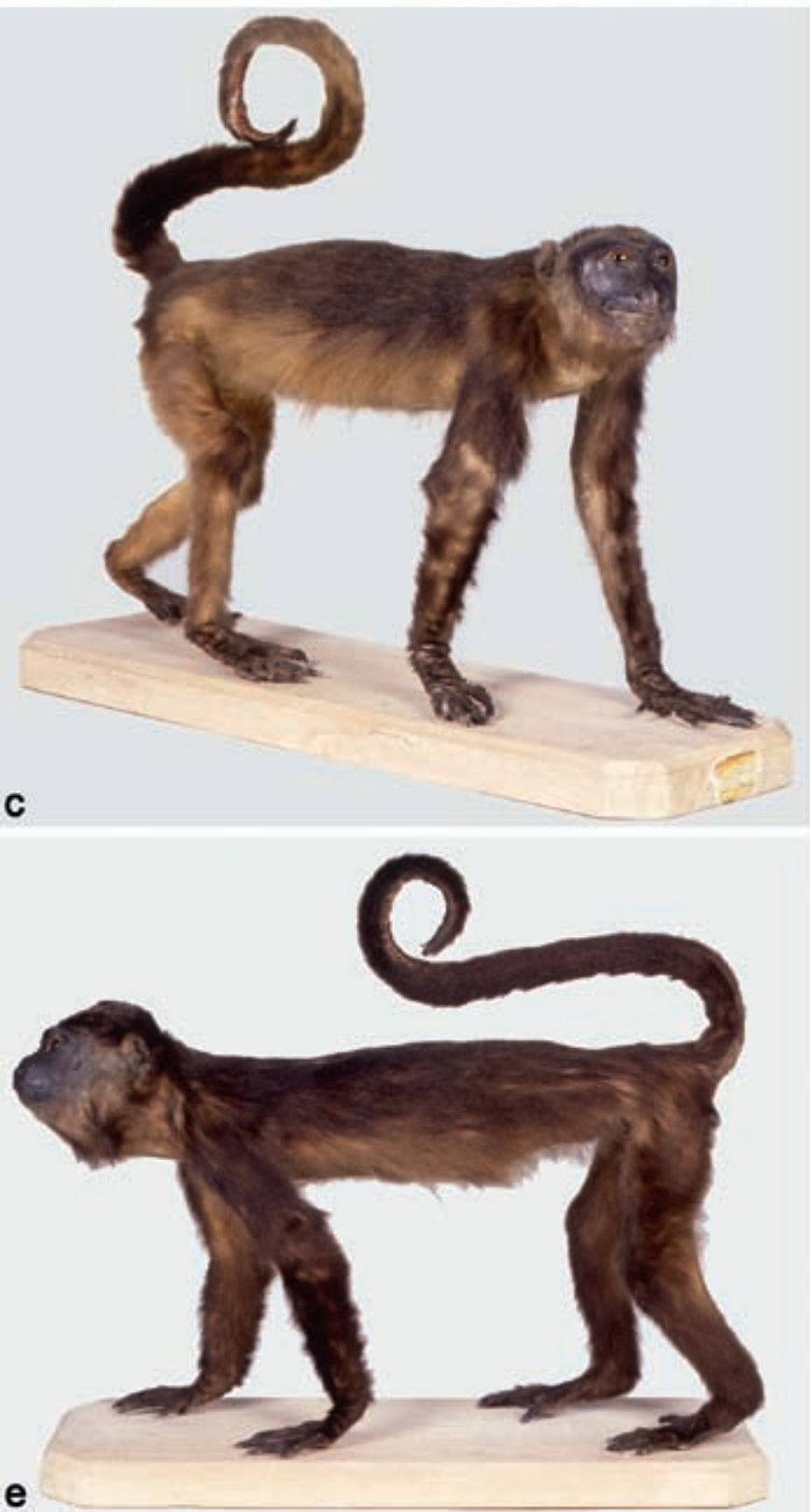
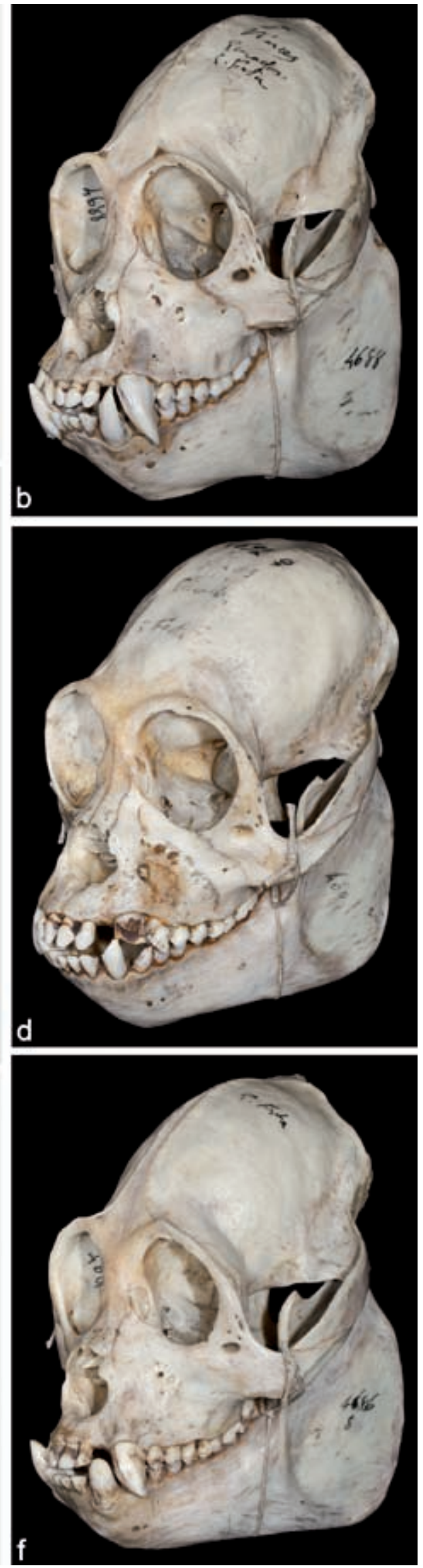

Fig. 1 - Type series of Alouatta palliata aequatorialis: a-b) Lectotype Male MZUT T 33, mount (a) and skull (b); c-d) Paralectotypes Female MZUT T 31, mount (c) and skull (d); e-f) Paralectotypes Male MZUT T 32, mount (e) and skull (f). 


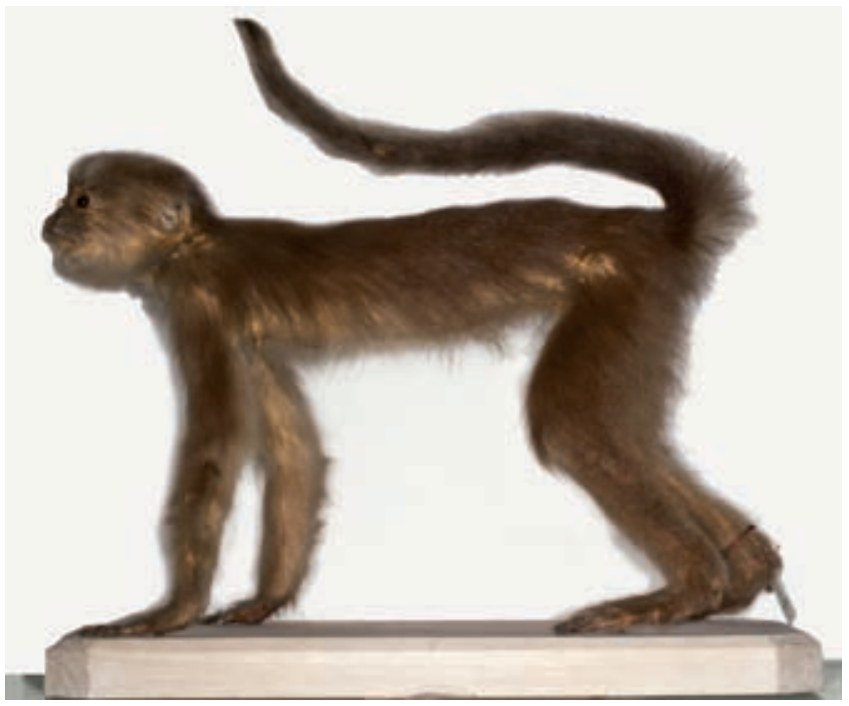

Fig. 2 - Type-specimen of the variety brunnea of Cebus albifrons cuscinus (MZUT T 57).

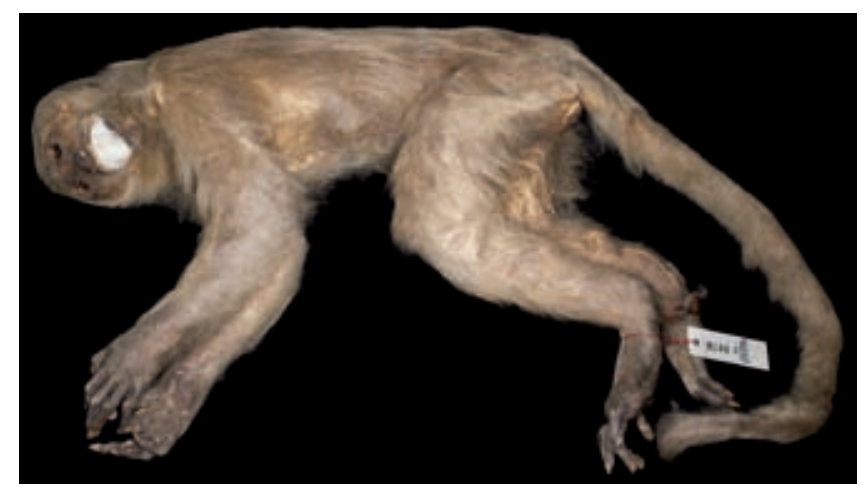

Fig. 3 - Skin of Sapajus flavius (MZUT T 76). This is the first specimen recorded in a European museum.

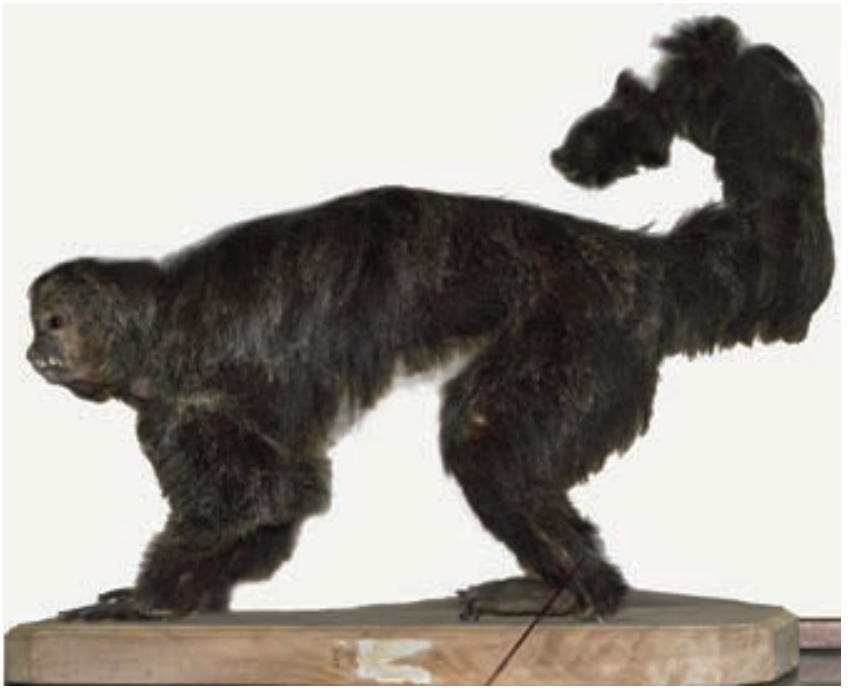

Fig. 4 - Mounted specimen of Pithecia pissinatti (MZUT T 81), species only recently described (Marsh, 2014).

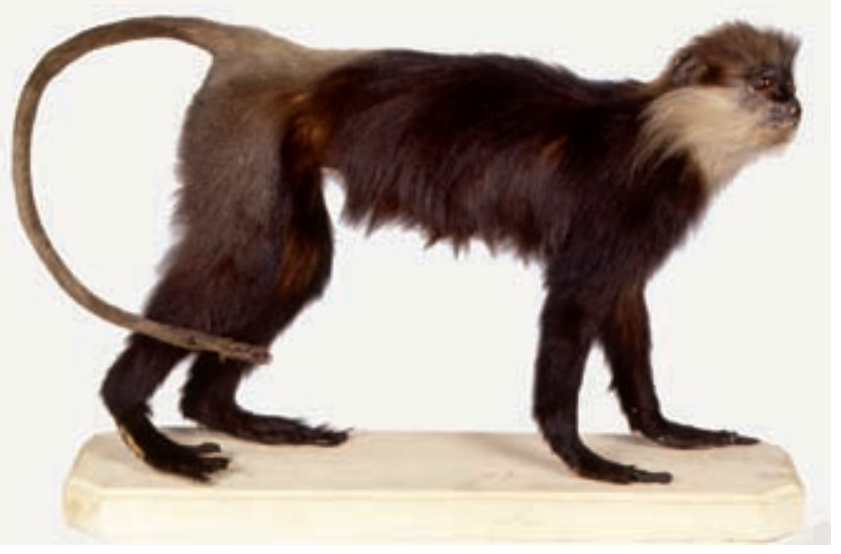

Fig. 5 - Mounted specimen of Semnopithecus vetulus (MZUT T 93) collected in 1885, two years before the formal description of Cercopithecus mitis boutourlinii, the species to which this specimen was referred in the original catalogues and labels.

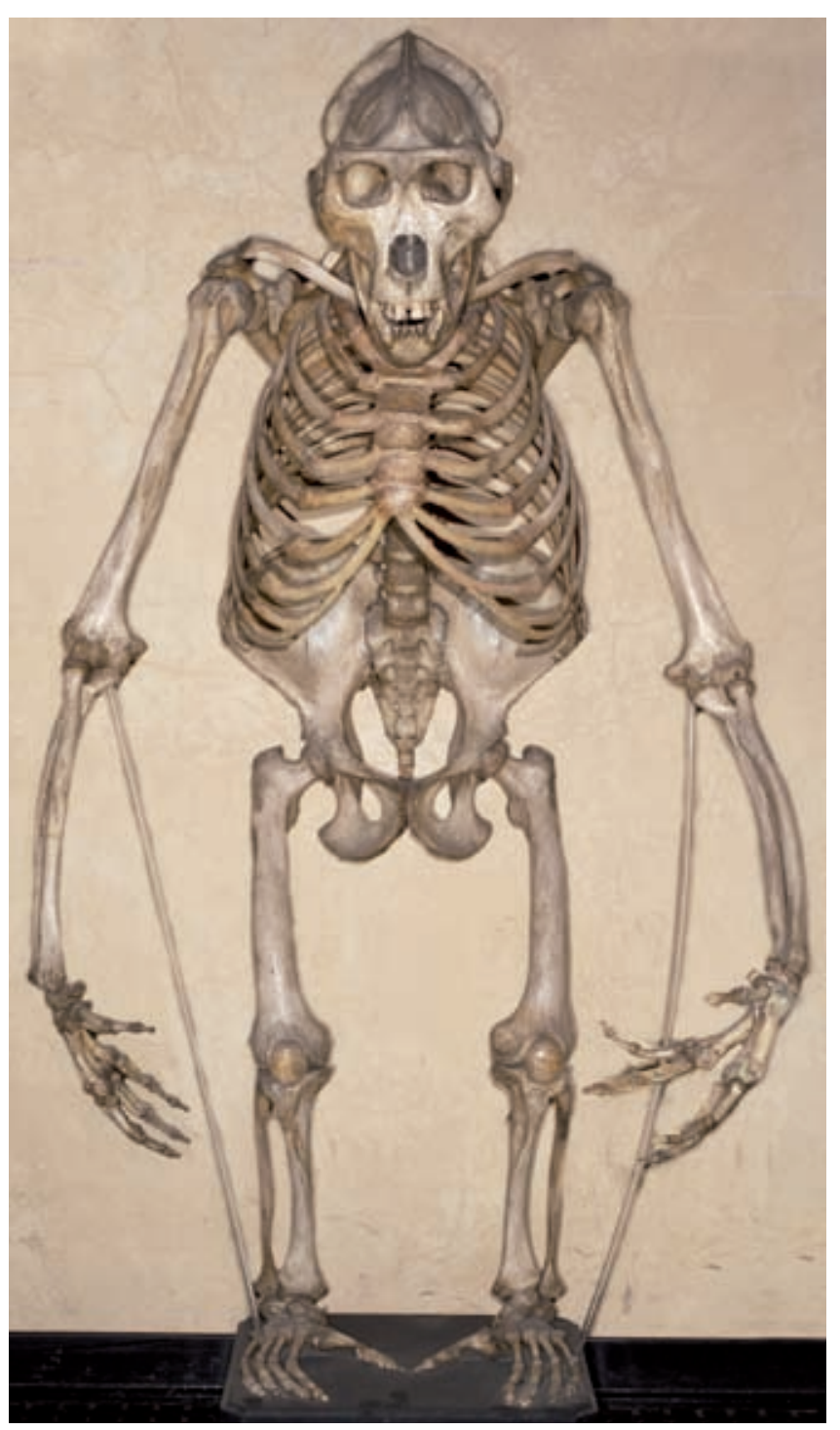

Fig. 6 - Complete skeleton of an adult male of Gorilla gorilla (MZUT $\mathrm{T}$ 323), the first to reach an Italian museum. This specimen arrived in Torino in 1862 and was most likely presented to the public by F. De Filippi in 1864. 


\section{REFERENCES}

Andreone F., Gavetti E. \& Bovero S., 2007 - Revised catalogue of the herpetological collection in Turin University. 2. Chelonia and Crocodylia. Bollettino del Museo Regionale di Scienze Naturali, Torino, 24 (2): 301-347.

Ardito G. \& Bigatti M.P., 2006 - Le Collezioni Primatologiche Torinesi. In: Le Collezioni Primatologiche Italiane. Bruner E. \& Gippoliti S. (a cura di). Istituto Italiano di Antropologia, Roma: 203-216.

Bigatti M.P., Bovero S., Gavetti E., Crovella S. \& Ardito G., 2011 - Alouatta palliata aequatorialis Festa, 1903: analysis of the typical material housed in the primatological collection of Turin University. Bollettino del Museo Regionale di Scienze Naturali, Torino, 28 (1): 9-19.

Camerano L., 1896 - Note zoologiche: 7. Di un cranio di Cercopithecus ruber con dentatura anomala. Bollettino dei Musei di Zoologia e Anatomia Comparata della Regia Università di Torino, 11 (251): 1-3.

Camerano L., 1909a - Ricerche intorno al Colobus occidentalis Rochebr e ad altre specie affini. In: Il Ruwenzori. Parte scientifica. Risultati delle osservazioni e studi compiuti sul materiale raccolto dalla spedizione. Volume I. Zoologia. Botanica. Hoepli, Milano: 1-66.

Camerano L., 1909b - Osservazioni intorno al Cercopithecus ascanias Aud. subsp. schmidti Matsch. In: Il Ruwenzori. Parte scientifica. Risultati delle osservazioni e studi compiuti sul materiale raccolto dalla spedizione. Volume I. Zoologia. Botanica. Hoepli, Milano: 67-73.

Crovella S., Andreone F. \& Moggi-Cecchi J., 1989 - La Collezione Primatologica del Dipartimento di Biologia Animale dell'Università di Torino. Proceedings of the $6^{\text {th }}$ National Congress of the Association Alessandro Ghigi. Museo Regionale di Scienze Naturali di Torino: $275-278$.

Crovella S. \& Passerin D'entrèves P., 1990 - Alouatta palliata aequatorialis, Festa, 1903: ridescrizione e definizione del materiale tipico. Atti Accademia delle Scienze di Torino, 124 (5-6): 208-216.

De Oliveira M.M. \& Langguth A., 2006 - Rediscovery of Marcgrave's capuchin monkey and designation of a neotype for Simia flavia Schreber, 1774 (Primates, Cebidae). Boletim do Museu Nacional Rio de Janeiro, Zoologia, 523: 1-16.

Festa E., 1903 - Viaggio del Dr. Enrico Festa nel Darien, nell'Ecuador e regioni vicine. Mammiferi, 1. Primates. Bollettino dei Musei di Zoologia ed Anatomia Comparata della $R$. Università di Torino, 18 (435): 1-9.

Festa E., 1909 - Nel Darien e nell'Ecuador. Diario di viaggio di un naturalista. UTET, Torino.

Gavetti E. \& Andreone F., 1993 - Revised catalogue of the herpetological collection in Turin University. 1. Amphibia. Museo Regionale di Scienze Naturali, Torino, Cataloghi 10.

Gentili S., Mottura A. \& Rook L., 1998 - The Italian fossil primate record: recent finds and their geological context. Geobios, 31 (5): 675-686.

Giovanardi S., 2014 - Le varianti morfologiche dei crani di primati non umani: un'analisi della Collezione
Osteologica dell'Università di Torino. Thesis, Torino University.

Giglioli E.H., 1887 - Nota intorno a una nuova specie di Cercopiteco del Kaffa (Africa Centrale). Zoologischer Anzeiger, 10 (261): 509-510.

Giglioli E.H., 1888 - Nota intorno agli animali vertebrati raccolti dal conte Boutourline e dal dott. Travesi ad Assab e nello Scioà negli anni 1884-87. Annali del Museo Civico di Storia Naturale di Genova, 6: 5-73.

Gippoliti S., 2009 - Da Antinori a Zavattari: uno sguardo alle collezioni primatologiche italiane e al loro ruolo per la conservazione della biodiversità. Museologia Scientifica, Memorie, 4: 137-140.

Groves C., 2005 - Order Primates. In: Mammal Species of the World: A Taxonomic and Geographic Reference ( $3^{\text {rd }}$ ed.). Wilson D.E. \& Reader D.M. (eds.). Johns Hopkins University Press, Baltimore: 111-184.

Lastella S., Siori M.S., Ligabue Stricker F. \& Micheletti Cremasco M., 2012 - The Primate Ear Bones Collection of the University of Turin: Revision and Improvement. Journal of Biological Research, 85 (1): 302-305.

Marsh L.K., 2014. A taxonomic revision of the Saki monkeys, Pithecia Desmarest, 1804. Neotropical Primates, 21: 1-163.

Maschetti G., Muti M. \& Passerin D’Entreves P., 1988 Serragli e Menagerie in Piemonte nell'Ottocento sotto la Real Casa Savoia. U. Allemandi \& C. Ed., Torino.

Mittermeier R.A., Rylands A.B. \& Wilson D.E., 2013 Handbook of the Mammals of the World. Volume 3. Primates. Lynx, Barcelona.

Mottura A. \& Ardito G., 1992 - Observations on the Turin specimen of Mesopithecus pentelici (Wagner, 1839). Human Evolution, 7 (1): 67-73.

Mottura A. \& Gentili S., 2006 - Cranio-Mandibular Biometrics and Skull Maturation of Macaca sylvanus L., 1758. Human Evolution 21: 223-239.

Rylands A.B., Kierulff C.M. \& Mittermeier R.A., 2005 Notes on the taxonomy and distributions of the tufted capuchin monkeys (Cebus, Cebidae) of South America. Lundiana, 6 (Supplement): 97-110.

Salvadori T. \& Festa E., 1899 - Viaggio del Dr. Enrico Festa nell'Ecuador. Uccelli, parte prima: Passeres oscines. Bollettino dei Musei di Zoologia ed Anatomia Comparata della R. Università di Torino, 15 (357): 1-31.

Sclater P.L., 1893 - Additional notes on the monkeys of the genus Cercopithecus. Proceedings Zoological Society London: 441-444.

Vanni S., Agnelli P. \& Azzaroli M.L., 1990 - Cataloghi del Museo Zoologico "La Specola" dell'Università di Firenze. 9. Mammalia: Tipi. Atti Società Toscana di Scienze Naturali, Memorie, Serie B, 96: 231-238.

Visalberghi E. \& Valente M., 1997 - "Collezioni invisibili” Primates. Museo Regionale di Scienze Naturali, Torino.

Vuch J., Siori M.S., Bigatti M.P., Segat L., De Fabrizio G. \& Crovella S., 2013 - Analysis of the beta globin gene in DNA of suspected thalassemic great apes. Genetics and Molecular Research, 12 (2): 1731-1739. 
SYSTEMATIC CATALOGUE

Order PRIMATES Linnaeus, 1758

Family LEMURIDAE Gray, 1821

Genus Eulemur Simons \& Rumpler, 1988

Eulemur albifrons É. Geoffroy Saint-Hilaire, 1796

MZUT T $3 \quad(56$, M3)

MZUT T $20 \quad(55, \mathrm{M} 2)$ w.d., 9 Nov 1827, Tournaire exc., dead in captivity

MADAGASCAR, 1823, Dupont don.
1 Mount

1 Mount

1 Mount

1 Skull

1 Mount

1 Skull

1 Mount 1 partial Skeleton

1 Mount

MADAGASCAR., 1901, dead in captivity

MADAGASCAR, 1863, Bonomi don.

1 Mount

1 Skull

1 partial Skeleton

Genus Lemur Linnaeus, 1758

Lemur catta Linnaeus, 1758

MZUT T $7 \quad$ (919, M1)

(MACUT 76)

MZUT T 29

MZUT T $379 \quad$ (1860)

(MACUT 6039)

MZUT T 335 (MACUT 3482)

MADAGASCAR, 1863, from Verreaux

1 Mount

1 Skull

w.d., 3 Jul 1870

w.d., 1904, purchased

1 Mount

1 Skin

1 Skeleton

w.d., 1896, purchased from Crivelli

1 Skull

Genus Varecia Gray, 1863

Varecia variegata (Kerr, 1792)

MZUT T $10 \quad$ (1314)

MADAGASCAR, 1890, Peracca don.

1 Mount

Family LORISIDAE (Gray, 1821)

Genus Nycticebus É. Geoffroy Saint-Hilaire, 1812

Nycticebus bengalensis (Lacépède, 1800)

MZUT T $21 \quad$ (1669, M12)

MYANMAR, 1898, Bertoldo don.

1 Mount

Nycticebus javanicus É. Geoffroy Saint-Hilaire, 1812

MZUT T $18 \quad$ (57, M14)

MZUT T 19 (990, M13) w.d., Sept 1830, Leadbeater don. 1 Mount

INDONESIA, Giava, 1867, from De Filippi during M.H.S. 1 Mount Magenta exped. 
Family GALAGIDAE (Gray, 1825)

Genus Galago É. Geoffroy Saint-Hilaire, 1796

Galago senegalensis É. Geoffroy Saint-Hilaire, 1796

MZUT T $17 \quad(58$, M10)

MZUT T $1 \quad(638$, M11)

Galago sp.

MZUT T 388

(MACUT 7008)

(MACUT 7009)
SENEGAL, Nov 1831, Bonomi don.

SENEGAL, Jul 1841, purchased from Caffer

SOMALIA, Bardera, Jun 1910, Testa Fochi don.

SOMALIA, Bardera, Testa Fochi don.
1 Mount

1 Mount

1 Skeleton

1 Skeleton

Genus Otolemur Coquerel, 1859

Otolemur crassicaudatus É. Geoffroy Saint-Hilaire, 1812

MZUT T $2 \quad$ (873, M9)

AFRICA, 1857, De Filippi don.

1 Mount

Infraorder PLATYRRHINI É. Geoffroy Saint-Hilaire, 1812

Family CEBIDAE Gray, 1831

Genus Callimico Miranda-Ribeiro, 1912

Callimico goeldii (Thomas, 1904)

MZUT T 349 (MACUT 4164) w.d.

1 Skeleton

Genus Callithrix Erxleben, 1777

Callithrix jacchus (Linnaeus, 1758)

MZUT T 353

(MACUT 4267)

w.d., Adult

1 Skeleton

Callithrix sp.

MZUT T 220

(MACUT 5419,
$5420)$

w.d., 1902

w.d., 16 Sept 1829

w.d., 12 Sept 1829

MZUT T 307

MZUT T 395

(MACUT 7337)

w.d., Dead in captivity

BRAZIL, 1915, Bay don., dead in captivity

BRAZIL, 1916, Bonero don. Juvenile
1 Skeleton

1 Skull

1 partial Skull

1 partial Skull

1 Skeleton

1 Skeleton

1 Skull

Genus Mico Lesson, 1840

Mico melanurus (É. Geoffroy Saint-Hilaire, 1812)

$\begin{array}{ll}\text { MZUT T } 238 & \text { (1707) } \\ & \text { (MACUT 5236) }\end{array}$

BRAZIL, Mato Grosso, Urucum, 1902, Carcano don.

1 Skin

1 Skull

\section{Genus Leontopithecus Lesson, 1844}

Leontopithecus rosalia (Linnaeus, 1766)

MZUT T 22

(2521, M27)

(MACUT 2043)

MZUT T 23

(52, M28)

MZUT T 326

(MACUT 63)

MZUT T 331

(MACUT 2088)

MZUT T 394
BRAZIL, 1902, Bocca don.

w.d.

w.d., 1883, from Bosi

w.d.

w.d., Dead in captivity. Juvenile
1 Mount

1 Mount

1 Skull

1 Skeleton

1 Skeleton 
Genus Saguinus Hoffmannsegg, 1807

Saguinus geoffroyi (Pucheran, 1845)

$\begin{array}{ll}\text { MZUT T 5 } & (506) \\ \text { MZUT T 24 } & (\text { (1424, M29) } \\ & \text { (MACUT 3776) } \\ \text { MZUT T 241 } & \text { (MACUT 3775) }\end{array}$

Central and South America

1 Mount

1896, purchased from Bajnotti

1 Skull

PANAMA, Darien, Rio Cianati 1 Mount

1895, Festa exped. 1 Skull

PANAMA, Darien, Pita Lagoon, 1895, Festa don. Cited in 1 Skull

Saguinus midas (Linnaeus, 1758)

MZUT T $25 \quad$ (47, M30)

South America, 1819, from Bullock 1 Mount

MZUT T $26 \quad(48$, M31)

FRENCH GUIANA, Cajenna, 25 Jul 1823, from MNHN Paris 1 Mount

Saguinus niger (É. Geoffroy Saint-Hilaire, 1803)

MZUT T 28 (2761, M33)

Saguinus nigricollis (Spix, 1823)

MZUT T 27 (817, M32)

Cebus albifrons (Humboldt, 1812)

$\begin{array}{ll}\text { MZUT T 59 } & (1654, \text { M64) } \\ & \text { (MACUT 4690) } \\ \text { MZUT T 60 } & (35, \text { M65) } \\ \text { MZUT T 66 } & (1653, \text { M60) } \\ \text { MZUT T 233 } & \text { (MACUT 4687) }\end{array}$

MZUT T 383 (MACUT 6694) w.d., 27 May 1927, Molinar don., dead in captivity

1 Mount

MEXICO, From Franck

[Locality probably wrong]. [Labeled as $S$. ursula]

\section{Genus Cebus Erxleben, 1777}

ECUADOR, Rio Peripa forests, 1897, Festa exped. 1 Mount

1 Skull

South America, 1811, Borelli exped. Juvenile 1 Mount

ECUADOR, Vinces, 1897, Festa exped. Juvenile 1 Mount

ECUADOR, Vinces, 1897, Festa exped. Juvenile, Cited in 1 Skull

Festa (1903)

[Labeled as Sapajus sp.]

w.d., 1906, from Navaresio

1 Skull

Cebus albifrons cuscinus (Thomas, 1901)

MZUT T $57 \quad$ (1655, M61)

MZUT T 58

(1652, M62)

(MACUT 4689)

MZUT T 63 (1498, M63)

(MACUT 3976)

Cebus capucinus (Linnaeus, 1758)

MZUT T 70

(1414, M74)

(MACUT 3750)

MZUT T 71

(1433, M75)

(MACUT 3817)

MZUT T $72 \quad$ (1434, M76)

(MACUT 3811)

MZUT T 73

(1432, M77)

(MACUT 3816)

MZUT T $74 \quad$ (384, M78)

MZUT T $280 \quad$ (MACUT 3781)

MZUT T $296 \quad$ (1384)

(MACUT 3576)

MZUT T 299
ECUADOR, Granadillas, Jul 1897, Festa exped., Cited in Fe- 1 Mount sta (1903: p. 8) as "var. brunnea"

[Type-specimen of the variety]

ECUADOR, Vinces, Sept 1897, Festa exped. 1 Mount

ECUADOR, Vinces, Sept 1897, Festa exped. Adult 1 Mount

1 Skull

PANAMA, Darien, Pita Lagoon, 1895, Festa exped. Juvenile 1 Mount

1 Skull

PANAMA, Darien, Pita Lagoon, 1895, Festa exped. Adult 1 Mount

1 Skull

PANAMA, Darien, Punta de Sabana, 1895, Festa exped. Adult 1 Mount

1 partial skeleton

PANAMA, Darien, Pita Lagoon, 1895, Festa exped. Adult 1 Mount

1 Skull

w.d.

1 Mount

PANAMA, Darien, Pita Lagoon, 1895, Festa exped. 1 Skull

PARAGUAY, Jul 1893, Borelli exped. 1 Skin

[Species probably wrong] 1 Skull

w.d.

1 Skull 
$\begin{array}{ll}\text { MZUT T } 340 & \text { (MACUT 3625) } \\ \text { MZUT T } 355 & \text { (MACUT 4461) }\end{array}$

Cebus olivaceus Schomburgk, 1848

MZUT T $75 \quad$ (681, M79)
PANAMA, Darien, Pita Lagoon, 1895, Festa exped. Adult 1 Skull

BRAZIL, Mato Grosso, Curumbà, 1899, Borelli exped. Adult 1 Skull [Species probably wrong]

Central and South America, Jan 1844, from Poisson, dead in 1 Mount captivity
Sapajus apella (Linnaeus, 1758)

MZUT T $67 \quad(667$, M71)

MZUT T $68 \quad(1709$, M72)

(MACUT 5122)

MZUT T $69 \quad(1710$, M73)

(MACUT 5123)

MZUT T $165 \quad$ (29, M70)
Genus Sapajus (Kerr, 1792)

Central and South America, 1853, from D'Olivieri 1 Mount

BRAZIL, Mato Grosso, Urucum, 1901, Carcano don. 1 Mount [Probably S. libidinosus]

BRAZIL, Mato Grosso, Urucum, 1901, Carcano don. [Probably S. libidinosus]

Central and South America, 28 Jan 1829, Seyssel don.
1 partial Skull

1 Mount

1 Skull

1 Mount

Sapajus apella fatuellus (Linnaeus, 1766)

MZUT T 236 (MACUT 3459)

MZUT T 247 (MACUT 58)

MZUT T 273 (MACUT 2344)

MZUT T 334 (MACUT 3455)

MZUT T 337 (MACUT 3538)

MZUT T 342 (MACUT 3747)

Sapajus flavius (Schreber, 1774)

MZUT T $76 \quad$ (2784, M80)

Sapajus nigritus (Goldfuss, 1809)

MZUT T 64 (637, M66)

MZUT T $65 \quad(33$, M67)

Sapajus robustus (Kuhl, 1820)

MZUT T 62 (232, M69)

\section{Sapajus sp.}

MZUT T 61

(1317, M68)

MZUT T 77

(1037, M81)

MZUT T 259

(MACUT 7721)

MZUT T 276

(MACUT 7719)

MZUT T 279

MZUT T 401

MZUT T 402

(MACUT 7720)

(MACUT 7724)

w.d., 1896, purchased from Crivelli. Adult

1 Skull

w.d.

1 Skull

PARAGUAY, 1896, purchased from Crivelli

1 Skull

w.d., 1896, purchased from Crivelli. Adult

1 Skull

w.d., 5 Jan 1829, Masserino don., dead in captivity. Adult

1 Skull

ARGENTINA, 1895, purchased from Crivelli. Juvenile

1 Skeleton

ARGENTINA, 1911, Argentina Exibition Comitee don. [Labeled as Cebus sp.]

1 Skin

BRAZIL, Rio de Janeiro, Serra dos Órgãos, Sept 1839, Caffer 1 Mount in M.H.S. Regina exped. Immature

w.d., Oct 1826, Bonafous don., dead in captivity

1 Mount

SURINAME, 1845, from Franck

[Labeled from Guyana, locality probably wrong]

1 Mount

ECUADOR, 1890

1 Mount

[Labeled as S. nigritus]

w.d., 1869, Torino Zoological Garden don.

1 Mount

BRAZIL, 1916, Bovero don. Adult

1 Skull

BRAZIL, Bovero don.

1 partial Skull

w.d.

BRAZIL, 1916, Bovero don.

1 partial Skull

1 Skull

1 Skull

Genus Saimiri Voigt, 1831

Saimiri boliviensis (I. Geoffroy Saint-Hilaire \& Blainville, 1834)

MZUT T $82 \quad$ (1488, M89)

(MACUT 3959)
ECUADOR, Rio Santiago Valley, 1897, Festa exped., Cited in 1 Mount

Festa (1903: p. 7) as S. sciureus 
Saimiri sciureus (Linnaeus, 1758)

$\begin{array}{ll}\text { MZUT T } 83 & (337, \text { M90) } \\ \text { MZUT T } 84 & (36, \text { M91) } \\ \text { MZUT T } 325 & \text { (MACUT 61) }\end{array}$
Central and Southern America, 1853, D'Olivieri don.

1 Mount FRENCH GUIANA, Cajenna, 25 Jul 1823, from MNHN Paris 1 Mount w.d., 1853, D’Olivieri don.

1 Skull

Family AOTIDAE Elliot, 1913

Genus Aotus Illiger, 1811

Aotus azarae (Humboldt, 1811)

MZUT T $53 \quad$ (2212, M57)

(MACUT 7017)

PARAGUAY, Alto Paraguay, Fuerte, Olimpo, 1897, Festa don. 1 Mount [Labeled as A. trivirgatus roberti]

1 Skull

MZUT T 169 (1316, M55)

BOLIVIA, 1890

1 Mount

Aotus lemurinus (I. Geoffroy Saint-Hilaire, 1843)

MZUT T $52 \quad$ (879, M56)

BRAZIL, 1857, purchased in Paris by De Filippi

1 Mount

Aotus trivirgatus (Humboldt, 1811)

MZUT T 6

VENEZUELA, Orinoco, 1851, from Franck

1 Mount

Family PITHECIIDAE Mivart, 1865

Genus Callicebus Thomas, 1903

Callicebus cinerascens (Spix, 1823)

MZUT T $79 \quad$ (442, M83)

CHILE , 1851, from Franck

[locality probably wrong]. [Labeled as C. donacophilus]

1 Mount

Callicebus donacophilus (d'Orbigny, 1836)

MZUT T 78 (666, M82)

BOLIVIA, 1853, from D’Olivieri. Adult

1 Mount

(MACUT 64)

1 Skull

Genus Cacajo Lesson, 1840

Cacajo calvus (I. Geoffroy Saint-Hilaire, 1847)

MZUT T $54 \quad$ (24, M59)

BRAZIL, Rio Blanco, 1846, Ghigliani exped.

1 Mount

Genus Chiropotes Lesson, 1840

Chiropotes chiropotes (Humboldt, 1811)

MZUT T 167 (326, M84)

GUYANA

1 Mount

Genus Pithecia Desmarest, 1804

Pithecia monachus (É. Geoffroy Saint-Hilaire, 1812)

MZUT T $166 \quad$ (17, M87)

Pithecia pissinatti Marsh, 2014

MZUT T $81 \quad$ (97, M88)
SURINAME, Jan 1845, from Franck

[Labeled as Guyana, locality probably wrong]

BRAZIL, Rio Madeira, 1846, Ghigliani exped.

[Labeled as P. monachus]

Family ATELIDAE Gray, 1825

Genus Alouatta Lacépède, 1799

Alouatta caraya (Humboldt, 1812)

MZUT T $30 \quad$ (1382, M35)

(MACUT 3506)
PARAGUAY, Ajos, Jul 1893, Borelli exped.

1 Mount

1 Skull 
MZUT T 44

(1383, M52)

(MACUT 3523)

MZUT T 333 (MACUT 3452)

Alouatta guariba (Humboldt, 1812)

MZUT T 13 (634)

Alouatta palliata (Gray, 1849)

MZUT T 38 (1430, M44)

(MACUT 3810)

MZUT T $39 \quad$ (294, M45)

MZUT T $343 \quad$ (1415)

(MACUT 3751)
PARAGUAY, Rio Apa, Aug 1893, Borelli exped.

1 Mount

1 Skull

w.d., 1896, purchased from Crivelli

1 Skull

BRAZIL Rio de Janeiro, Serra dos Órgãos. Sept 1839, Caffer 1 Mount in M.H.S. Regina exped.

PANAMA, Darien, Rio Lara, 1895, Festa exped.

1 Mount

1 Skull

PANAMA, Darien, Rio Cianati forest, 6 Jun 1895, Festa ex- 1 Mount ped., Cited in Festa (1903)

[Labeled as Alouatta sp.]

PANAMA, Darien, Pita Lagoon, 1895, Festa exped. Adult 1 Skin

1 Skull

Alouatta palliata aequatorialis Festa, 1903

$\begin{array}{ll}\text { MZUT T } 31 & \text { (1658, M36) } \\ & \text { (MACUT 4692) }\end{array}$

MZUT T $32 \quad(1657$, M37)

(MACUT 4686)

MZUT T $33 \quad$ (1656, M38)

(MACUT 4688)

MZUT T 34

(1659, M39)

(MACUT 4693)

ECUADOR, Vinces, 1897, Festa exped. F.

[Paralectotype here selected]

1 Mount

1 Skull

ECUADOR, Vinces, 1897, Festa exped. M. 1 Mount

[Paralectotype here selected]

1 Skull

ECUADOR, Vinces, 1897, Festa exped. M. 1 Mount

[Lectotype here selected]

1 Skull

ECUADOR, Vinces, 1897, Festa exped. Juvenile

[Paralecto-type here selected]

1 Mount

1 Skull

Alouatta seniculus (Linnaeus, 1766)

MZUT T $36 \quad$ (286, M41)

(MACUT 55)

BOLIVIA, 1853, from D'Olivieri.

[Probably A. sara Elliot, 1910]

1 Mount

1 Skull

BOLIVIA, 1853, from D'Olivieri. Immature

1 Mount

[Probably A. sara Elliot, 1910]

w.d., 1854. Adult

1 Skull

w.d., 1854. Adult

1 Skull

BRAZIL, 1822, Dupont don.

[Labeled as A. seniculus arctoidea]

1 Mount

1 Skull

MZUT T 312 (MACUT 35)

w.d., 1877, from Torino Zoological Garden, dead in captivity. 1 Skull Adult

w.d.

1 Skull

w.d.

Genus Ateles É. Geoffroy Saint-Hilaire, 1806

Ateles belzebuth É. Geoffroy Saint-Hilaire, 1806)

MZUT T $40 \quad(1319$, M47)

MZUT T $41 \quad$ (1487, M46)

(MACUT 3958)

MZUT T $42 \quad(1635$, M48)

MZUT T $43 \quad(1661$, M49)

(MACUT 4837)

MZUT T 239 (MACUT 3879)

MZUT T 346 (MACUT 3886)
BRAZIL, 1890, S.A. Duca di Genova don. 1 Mount

ECUADOR, San Josè, May 1897, Festa exped. Immature 1 Mount

1 Skull

ECUADOR, Rio Santiago Valley, Mar 1897, Festa exped. Adult 1 Mount

ECUADOR, Rio Santiago Valley, Mar 1897, Festa exped. Im- 1 Mount mature

1 Skull

ECUADOR, Rio Santiago Valley, 1896, Festa exped. 1 Skull

ECUADOR, Rio Santiago Valley, 1896, Festa exped. Adult, Ci- 1 partial Skull ted in Festa (1903: p. 4) as A. variegatus,

[Labeled as Ateles sp.] 
MZUT T 372 (MACUT 5526)

MZUT T 373 (MACUT 5527)

Ateles fusciceps Gray, 1866

MZUT T $46 \quad$ (626, M54)

(MACUT 3504)

Ateles geoffroyi Kuhl, 1820

MZUT T 45

MZUT T $168 \quad(836$, M51)

MZUT T $170 \quad$ (955, M50)

Ateles paniscus (Linnaeus, 1758)

MZUT T $171 \quad$ (27, M196)

(MACUT 50)

Ateles sp.

MZUT T $114 \quad$ (28, M122)
ECUADOR, Rio Santiago Valley, 1897, Festa exped., Cited in 1 Skull Festa (1903: p. 4) as A. variegatus

[Labeled as Sapajus apella]

ECUADOR, Rio Santiago Valley, 1897, Festa exped., Cited in 1 Skull Festa (1903: p. 4) as A. variegatus

[Labeled as Sapajus apella]

PANAMA, Darien, Rio Lara, 1895, Festa exped. Adult, Cited 1 Mount in Festa (1903) as A. ater

1 Skull

[Labeled as Ateles sp.]

w.d., 1869, Torino Zoological Garden don., dead in captivity 1 Mount [Labeled as Ateles sp.]

w.d., Juvenile

1 Mount

COLOMBIA, Jan 1865, from Bonomi

1 Mount

GUYANA, 1824, from MNHN Paris

1 Mount

1 Skull

w.d., Nov 1822, MNHN Paris don., dead in captivity

1 Mount

Genus Brachyteles Spix, 1823

Brachyteles arachnoides É. Geoffroy Saint-Hilaire, 1806

MZUT T 55 (M58)

MZUT T 305 (MACUT 37)

MZUT T 321 (MACUT 52)
ARGENTINA, 1911, Argentina Exibition Comitee don.

w.d., Adult

w.d., Adult
1 Mount

1 partial Skull

1 Skull

Genus Lagothrix É. Geoffroy Saint-Hilaire, 1812

Lagothrix lagotricha (Humboldt, 1812)

MZUT T 89 (283, M85)

BRAZIL, 1866, M.H.S. Magenta exped.

1 Mount

Lagothrix poeppigii Schinz, 1844

MZUT T 80

ECUADOR, Rio Santiago Valley, Festa exped., Cited in Festa 1 Mount (1903) as L. infumata

[Labeled as Lagothrix sp.]

MZUT T 258 (MACUT 4691)

ECUADOR, Rio Santiago Valley, Festa exped., Cited in Festa 1 Skull (1903: p. 4) as L. infumata

[Labeled as Platyrrhini indet.]

\section{PLATYRRHINI indet.}

MZUT T $216 \quad$ (MACUT 5395, w.d., 1902

1 Skeleton

5396)

MZUT T $240 \quad$ (MACUT 3847)

1896, from Bajnotti. Juvenile

1 Skull

MZUT T 248

(MACUT 54)

w.d.

1 Skull

1 Skull

Infraorder CATARRHINI É. Geoffroy Saint-Hilaire, 1812

Family CERCOPITHECIDAE Gray, 1821

Genus Cercocebus É. Geoffroy Saint-Hilaire, 1812

Cercocebus atys (Audebert, 1797)

MZUT T $85 \quad$ (1496, M92)

ITALY, Piemonte, Torino, 1898, dead in captivity. ? don. 1 Mount

(MACUT 4197) 


$\begin{array}{llll}\text { MZUT T } 86 & (1338, \text { M93) } & \text { w.d., } 1892 & 1 \text { Mount } \\ \text { MZUT T } 293 & \text { (MACUT 2228) } & \text { w.d., 1896, purchased from Crivelli } & 1 \text { Mount } \\ & & & 1 \text { Skull }\end{array}$

Cercocebus torquatus (Kerr, 1792)

$\begin{array}{ll}\text { MZUT T } 87 & \text { (2019, M94) } \\ & \text { (MACUT 6759) }\end{array}$

w.d., 1904, Bajnotti don., dead in captivity

1 Mount

1 Skull

Cercocebus sp.

MZUT T 399 (MACUT 7704)

DEM. REP. OF CONGO, 1916, Neri don. Adult

1 Skull

Genus Cercopithecus Linnaues, 1758

Cercopithecus ascanius katangae Lönnberg, 1919
MZUT T $107 \quad$ (2210, M115)
DEM. REP. OF CONGO, Katanga, 1911, purchased from Baj- 1 Mount notti
MZUT T $108 \quad$ (2209, M116)
DEM. REP. OF CONGO, Katanga, 1911, purchased from Baj- 1 Mount notti
MZUT T $109 \quad$ (2208, M117)
(MACUT 7079)
DEM. REP. OF CONGO, Katanga, 1911, purchased from Baj- 1 Mount notti
1 Skull

Cercopithecus ascanius schmidti Matschie, 1892
MZUT T 15
(2004)
UGANDA, Ruwenzori, 1906, S.A.R. Duca degli Abruzzi ex-
ped., Adult M. Cited in Camerano (1909b)
1 Mount
(MACUT 6804, 6803)
1 Skull
1 partial Skeleton

Cercopithecus cephus (Linnaeus, 1758)
MZUT T $91 \quad$ (820, M97)
GUINEA, Mar 1847, from Franck
[Locality probably wrong or possibly the ex Spanish Guinea, now Equatorial Guinea]

1 Mount

Cercopithecus diana (Linnaeus, 1758)

MZUT T $92 \quad$ (1326, M98) w.d., 1890

1 Mount

Cercopithecus mitis albogularis (Sykes, 1831)

MZUT T 265 (MACUT 39) w.d., Oct 1824, Fonseca don.

1 Skull

Cercopithecus mona (Schreber, 1774)

$\begin{array}{ll}\text { MZUT T } 94 & (1201, \text { M100) } \\ & \text { (MACUT 5369, } \\ & \text { 5370) }\end{array}$

MZUT T $251 \quad$ (MACUT 34)
ITALY, Piemonte, Torino, 23 Feb 1878, from Torino Zoologi- 1 Mount cal Garden, dead in captivity. Adult

1 Skull

1 Skeleton

ITALY, Piemonte, Torino, 1877, Torino Zoological Garden 1 Skull don., dead in captivity

Cercopithecus petaurista petaurista (Schreber, 1774)

MZUT T 95

(1499, M102)

w.d., 1898, purchased from Ghisolfi

1 Mount

MZUT T 96

(1422, M101)

(MACUT 3824)

w.d., 1895, Bertieri don.

1 Mount

1 Skeleton

Cercopithecus sp.

$\begin{array}{llll}\text { MZUT T } 112 & (1342, \text { M120) } & \text { w.d., 1892 } & 1 \text { Mount } \\ \text { MZUT T 113 } & (302, \text { M121) } & \text { w.d. Juvenile } & 1 \text { Mount } \\ \text { MZUT T 195 } & \text { (MACUT 4763) } & \text { w.d. } & 1 \text { Skeleton } \\ \text { MZUT T 197 } & \text { (MACUT 71) } & \text { w.d. } & 1 \text { Skeleton } \\ \text { MZUT T 235 } & \text { (MACUT 3544) } & \text { w.d. } & 1 \text { Skull } \\ \text { MZUT T 261 } & \text { (MACUT 32) } & \text { w.d. } & 1 \text { Skull } \\ \text { MZUT T 267 } & \text { (MACUT 3458) } & \text { w.d., 1896, purchased from Crivelli } & 1 \text { Skull } \\ \text { MZUT T 270 } & \text { (MACUT 3717) } & \text { w.d. } & 1 \text { Skull }\end{array}$




$\begin{array}{llll}\text { MZUT T 292 } & \text { (MACUT 4520) } & \text { w.d. } & 1 \text { Skull } \\ \text { MZUT T 300 } & \text { (MACUT 3897) } & \text { DEM. REP. OF CONGO, 1897, Gariazzo don. } & 1 \text { partial Skull } \\ \text { MZUT T 313 } & \text { (MACUT 40) } & \text { w.d. Adult } & 1 \text { partial Skull } \\ \text { MZUT T 365 } & \text { (MACUT 5334, } & \text { w.d., 1902. Adult } & 1 \text { Skull } \\ & 5335,5336,5337, & & 1 \text { partial Skeleton } \\ & 5338,5339,5340) & & 1 \text { Skull }\end{array}$

Chlorocebus aethiops (Linnaeus, 1758)

\begin{tabular}{|c|c|c|c|}
\hline MZUT T 88 & $(825$, M95) & $\begin{array}{l}\text { ITALY, Piemonte, Nichelino, Stupinigi, } 1 \text { Jan 1848, dead in } \\
\text { captivity }\end{array}$ & 1 Mount \\
\hline MZUT T 90 & $\begin{array}{l}(9, \text { M96) } \\
\text { (MACUT 3785) }\end{array}$ & $\begin{array}{l}\text { AFRICA, 1819, S.A. R. Principe di Carignano don., dead in } \\
\text { captivity }\end{array}$ & $\begin{array}{l}1 \text { Mount } \\
1 \text { Skull }\end{array}$ \\
\hline MZUT T 106 & $\begin{array}{l}(1035, \text { M113) } \\
\text { (MACUT 38) }\end{array}$ & $\begin{array}{l}\text { ITALY, Piemonte, Torino, } 16 \text { Aug } 1868 \text {, from Torino Zoologi- } \\
\text { cal, Garden, dead in captivity. Adult }\end{array}$ & $\begin{array}{l}1 \text { Mount } \\
1 \text { Skull }\end{array}$ \\
\hline MZUT T 184 & (MACUT 6083) & w.d., 1904 & 1 Skeleton \\
\hline MZUT T 314 & (MACUT 42) & w.d. Adult & 1 Skull \\
\hline MZUT T 369 & (MACUT 7388) & w.d., 4 Jan 1915 , dead in captivity. Juvenile & 1 Skeleton \\
\hline
\end{tabular}

Chlorocebus cynosuros (Scopoli, 1786)

MZUT T $56 \quad(6$, M104)

AFRICA, 11 Jan, Count Olivero di Roccabigliera, don., dead 1 Mount in captivity

MZUT T $97 \quad(8$, M103)

AFRICA, Aug 1831, dead in captivity

1 Mount

MZUT T 245 (MACUT 36)

w.d.

1 Skull

Chlorocebus pygerythrus (F. Cuvier, 1821) $\begin{array}{ll}\text { MZUT T } 99 & (1497, \text { M105) } \\ & \text { (MACUT 4194) }\end{array}$

ITALY, Piemonte, Torino, 1898, dead in captivity 1 Mount

MZUT T 253 (MACUT 3920)

BOTSWANA, 1897, Holub don. Juvenile

1 Skeleton

1 Skull

Chlorocebus sabaeus (Linnaeus, 1766)

MZUT T 102 (1103, M109)

MZUT T $103 \quad(2523$, M110)

(MACUT 7285)

MZUT T $104 \quad$ (880, M111)

MZUT T $105 \quad$ (1077, M112)

Chlorocebus sp.

MZUT T $111 \quad$ (1013, M119)
ITALY, Piemonte, Torino, 1874, from Torino Zoological Garden, dead in captivity

AFRICA, 1914, Bocca don., dead in captivity

ITALY, Piemonte, Torino, 1859, dead in captivity

ITALY, Piemonte, Torino, 1871, from Torino Zoological Garden, dead in captivity

ITALY, Piemonte, Torino, 1868, from Torino Zoological Gar- 1 Mount den, dead in captivity

[Labeled as Cercopithecus sp.]

\section{Genus Erythrocebus Trouessart, 1897}

Erythrocebus patas (Schreber, 1775)

MZUT T 98

(977, M106)

(MACUT 3835)

MZUT T $100 \quad(680$, M107)
ITALY, Piemonte, Torino, From Torino Zoological Garden, 1 Mount dead in captivity. Cited in Camerano (1896)

1 Skull

ITALY, Piemonte, Torino, Jan 1844, from Poisson, dead in 1 Mount captivity 
Genus Macaca Lacépède, 1799

Macaca assamensis McClelland, 1840

MZUT T $144 \quad$ (1113, M156)

CHINA, 1867, M.H.S. Magenta exped. M.

1 Mount

[Labeled as Macaca sp.]

Macaca fascicularis (Raffles, 1821)

MZUT T $120 \quad$ (12, M137)

MZUT T $121 \quad$ (1101, M138)

MZUT T 122 (1330, M139)

MZUT T 200 (MACUT 5225)

MZUT T 211 (MACUT 5375, 5376)

MZUT T 262 (MACUT 3356)

MZUT T 351 (MACUT 4193)

MZUT T 354

(MACUT 4425)

MZUT T 364

(MACUT 4722)

MZUT T 366

(MACUT 5352)

MZUT T 367

(MACUT 5371, 5372)

MZUT T 368 (MACUT 5373, 5374)

MZUT T 367

(MACUT 5401, 5402)
INDONESIA, Sunda Archipelago, 5 Feb 1829, Masserino don., dead in captivity. Adult

ITALY, Piemonte, Torino, 1874, from Torino Zoological Gar- 1 Mount den, dead in captivity

w.d., 1890

1 Mount

w.d., 1902, dead in captivity. Juvenile

w.d., 1902

1 Skeleton

1 Skull

1 Skeleton

w.d.

1 partial Skeleton

ITALY, Piemonte, Torino, 5 Feb 1829, from Moperino, dead in 1 Skeleton captivity

w.d., 1900, dead in captivity. Adult

1 Skeleton

w.d.

1 Skeleton

w.d.

1 Skeleton

w.d., 1902. Adult

1 Skull

1 Skeleton

w.d.

1 Skull

1 Skeleton

w.d.

1 Skull

1 Skeleton

Macaca mulatta (E.A.W. Zimmermann, 1780)

\begin{tabular}{|c|c|}
\hline MZUT T 119 & $(1320, \mathrm{M} 136)$ \\
\hline MZUT T 352 & (MACUT 4216) \\
\hline MZUT T 357 & $\begin{array}{l}\text { (MACUT 4465, } \\
4466)\end{array}$ \\
\hline MZUT T 358 & (MACUT 4486) \\
\hline MZUT T 361 & (MACUT 4583) \\
\hline MZUT T 362 & (MACUT 4584) \\
\hline IZUT T 404 & (MACUT 7788) \\
\hline
\end{tabular}

w.d., 1890

1 Mount

w.d., 1890

1 Skeleton

w.d., dead in captivity

1 Skeleton

w.d., 1900, dead in captivity

1 Hyoid bone

w.d.

1 Skeleton

w.d.

1 Skeleton

1 Skeleton

ITALY, Piemonte, Chivasso, 1916, dead in captivity

1 Skull

Macaca nemestrina (Linnaeus, 1766)

MZUT T $124 \quad(1202$, M142)

MZUT T $125 \quad(1251, \mathrm{M} 143)$

MZUT T $126 \quad(819$, M144)

MZUT T $127 \quad(1323$, M146)

MZUT T 132 (1213, M154)

MZUT T 192

(MACUT 4095)

MZUT T 205

(MACUT 65)

MZUT T 207
ITALY, Piemonte, Torino, 14 Feb 1878, from Torino Zoologi- 1 Mount cal, Garden, dead in captivity

INDONESIA, Sunda Archipelago, 12 Dec 1879, from Torino 1 Mount Zoological Garden, dead in captivity

INDONESIA, Java, Nov 1847, from Guillaume Compagnia 1 Mount Equestre, dead in captivity

w.d., 1890

1 Mount

ITALY, Piemonte, Torino, 1878, from Torino Zoological Gar- 1 Mount den, dead in captivity

[Labeled as Macaca sp.]

w.d., 1899

1 Skeleton

w.d.

1 Skeleton

w.d. 
MZUT T 208

MZUT T 277

(69)

MZUT T 285

MZUT T 286

MZUT T 315

MZUT T 322

MZUT T 330

(MACUT 1803)

MZUT T 356

(MACUT 4463)

MZUT T 360

(MACUT 4566)

MZUT T 363

(MACUT 4585)

MZUT T 378

(MACUT 5937)

Macaca nigra (Desmarest, 1822)

MZUT T 115 (2020, M123)

(MACUT 6762)

MZUT T $116 \quad$ (1389, M124)

MZUT T $117 \quad$ (1104, M125)

MZUT T $118 \quad(7$, M126)

Macaca ochreata (Ogilby, 1841)

MZUT T $380 \quad$ (1974, M141)

(MACUT 6234)

Macaca silenus (Linnaeus, 1758)

MZUT T 129 (11, M150)

Macaca sinica (Linnaeus, 1771)

MZUT T $101 \quad$ (1325, M108)

MZUT T $128 \quad$ (1329, M148)

MZUT T 376 (MACUT 5912)

MZUT T 377 (MACUT 5913)

MZUT T $381 \quad$ (1975)

(MACUT 6235)

Macaca sylvanus (Linnaeus, 1758)

$\begin{array}{ll}\text { MZUT T 130 } & (18, \mathrm{M} 151) \\ \text { MZUT T 131 } & (16, \mathrm{M} 152) \\ \text { MZUT T 154 } & (\mathrm{R} 1, \mathrm{~N} 2) \\ \text { MZUT T 264 } & \text { (MACUT 41) } \\ \text { MZUT T } 328 & \text { (MACUT 72) } \\ \text { MZUT T 329 } & \text { (MACUT 73) } \\ \text { MZUT T 374 } & \text { (MACUT 5642, } \\ & \text { 5643) }\end{array}$

MZUT T 393 (MACUT 7121) w.d., from Menagerie Bidel, dead in captivity

w.d., 1896

1 partial Skeleton

w.d.

1 Skull

1 Skull

w.d., from Festa 1 Skull

w.d., $1877 \quad 1$ Skull

NETHERLANDS, Amsterdam, Jan 1845, dead in captivity. 1 Skeleton Juvenile

w.d. Juvenile

1 Skeleton

w.d., dead in captivity

1 Skeleton

w.d., dead in captivity

1 Skeleton

w.d.

1 Skeleton

w.d

1 Skeleton

w.d., 1907, dead in captivity

1 Mount

1 Skeleton

ITALY, Liguria, Genova, 1893, dead in captivity. Juvenile 1 Mount

ITALY, Piemonte, Torino, 1874, from Torino Zoological Gar- 1 Mount den, dead in captivity

INDONESIA, Celebes Island, Aug 1825, Tournaire don., dead 1 Mount in captivity

w.d., dead in captivity

1 Skin

1 Skeleton

Oriental Indies, Jun 1831, Bonomi don., dead in captivity 1 Mount

w.d., 1890

[Labeled as Cercopithecus sp.]

w.d., 1890

w.d., 1904, Mosso don. Juvenile

1 Mount

1 Skeleton

w.d., 1904, Mosso don.

1 Skeleton

w.d., 1890, dead in captivity

1 Mount

1 Skeleton

w.d., 1827, Rolando don. Juvenile

1 Mount

w.d., 29 Jan 1829, Bandissero don. Immature

1 Mount

w.d.

[Labeled as Macaca sp.]

1 Mount

w.d.

1 Skeleton

w.d., 29 Jan 1829, Bandissero don. Adult

1 Skeleton

w.d.,

1827, Rolando don. Juvenile

1 Skeleton

w.d., 1903, purchase from heir of Cav. Cantù. Juvenile

1 Skull

1 Skeleton

w.d., 1911, Bocca don., dead in captivity

1 Skeleton 
Macaca sp.

MZUT T $110 \quad$ (1107, M118)

MZUT T 181

MZUT T 182

MZUT T 198

MZUT T 213

MZUT T 215

MZUT T 217

MZUT T 278

MZUT T 283

MZUT T 310

MZUT T 341
(MACUT 5353)

(MACUT 7296)

(MACUT 7919)

(MACUT 5417, 5418)

(MACUT 5389, 5390)

(MACUT 5399, 5400)

(MACUT 3784)

(MACUT 48)

(2675)

(MACUT 3719)
ITALY, Piemonte, Torino, 1874, from Torino Zoological Gar- 1 Mount den, dead in captivity [Labeled as Cercopithecus sp.]

w.d., 1902

1 Skeleton

w.d., 28 Mar 1914, from Baii

1 Skeleton

w.d.

1 Skeleton

w.d., 1902

1 Skull

1 Skeleton

w.d., 1902

1 Skull

1 Skeleton

w.d., 1902

1 Skull

1 Skeleton

w.d., 1896

1 Skull

ITALY, Piemonte, Bra, dead in captivity

1 Skull

MYANMAR, 1898, Bertoldo don.

1 Skin

w.d.

1 Skeleton

Genus Mandrillus Ritgen, 1824

Mandrillus leucophaeus (F. Cuvier, 1807)

MZUT T 332 (MACUT 3385)

w.d., Purchased from Crivelli. Juvenile

1 Skull

[Labeled as Papio sp.]

Mandrillus sphinx (Linnaeus, 1758)

MZUT T 143

w.d. Adult, $\mathrm{M}$

1 Mount

MZUT T $145 \quad$ (1339, M159)

w.d., 1892, Juvenile

1 Mount

MZUT T 179 (MACUT 70)

w.d., 1852, dead in captivity

1 partial Skeleton

MZUT T 344

(MACUT 3782)

w.d., 1896

[Labeled as Papio sp.]

1 Skull

Genus Miopithecus I. Geoffroy Saint-Hilaire, 1862

Miopithecus talapoin (Schreber, 1774)

MZUT T 289 (MACUT 33) w.d.

1 Skull

Genus Papio Erxleben, 1777

Papio anubis (Lesson, 1827)

MZUT T $162 \quad$ (1099, M161)

Papio hamadryas (Linnaeus, 1758)

$\begin{array}{ll}\text { MZUT T } 140 & (1327, \text { M165) } \\ \text { MZUT T } 141 & \text { (M166) }\end{array}$

MZUT T $142 \quad(1207$, M164)

MZUT T $163 \quad(1526$, M162)

(MACUT 4044)

MZUT T 194 (MACUT 7930, 7932)
ITALY, Piemonte, Torino, 10 Mar 1874, from Torino Zoologi- 1 Mount cal Garden, dead in captivity. Adult, M

w.d., 1890. Juvenile

1 Mount

ITALY, Piemonte, Torino, 1893, dead in captivity. Juvenile [Labeled as Papio sp.]

ITALY, Piemonte, Torino, 1878, from Torino Zoological Gar- 1 Mount den, dead in captivity

w.d., 1898, dead in captivity. Adult, M

w.d., 1898, dead in captivity. Adult
1 Mount 1 Skeleton

1 partial Skull 1 partial Skeleton 
MZUT T $304 \quad$ (2691)

MZUT T $348 \quad$ (MACUT 3947)

MZUT T $350 \quad$ (MACUT 4181)

MZUT T 359 (MACUT 4564)

Papio papio (Desmarest, 1820)

MZUT T $48 \quad(23$, M166)

(MACUT 4260)

MZUT T 234 (MACUT 3541)

Papio sp.

MZUT T 183

MZUT T 266

MZUT T 290

MZUT T 316

MZUT T 317

MZUT T 318
(MACUT 4094)

(MACUT 3560)

(MACUT 3457)

(MACUT 45)

(MACUT 46)

(MACUT 47)
ERITREA, Ghinda, 1899, Rossi don. M.

[Labeled as Papio sp.]

w.d., 1897, Cap. Muzioli from Perugia Museum don.

[Labeled as Papio sp.]

ERITREA, Massaua, Saganeiti, 1899, from Gentile. Adult

w.d., dead in captivity
1 Skin

1 partial Skull

1 Skull

1 partial Skull

1 Skeleton
ITALY, Piemonte, Torino, 16 Feb 1829, Masserino don., dead 1 Mount in captivity

1 Skeleton

w.d.

1 Skull

w.d., 1899

1 Skeleton

w.d.

w.d., 1896, purchased from Crivelli

1 Skull

1 Skull

w.d. Adult

1 Skull

1 Skull

[Possibly P. hamadryas]

w.d.

[Possibly Theropithecus gelada]

1 Skull

Genus Theropithecus (I. Geoffroy Saint-Hilaire, 1841)

Theropithecus gelada (Rüppell, 1835)

$\begin{array}{ll}\text { MZUT T } 16 \quad \text { (1992) } \\ & \text { (MACUT 6191) }\end{array}$
1 Mount

1 Skeleton

Colobus angolensis $\mathrm{P}$. Sclater, 1860

\begin{abstract}
MZUT T 137
(2205, M132)

(MACUT 7077)

MZUT T 392
\end{abstract}

(MACUT 7078) w.d., 1906, purchased from Bajnotti, dead in captivity

Colobus guereza Rüppell, 1835

MZUT T 222 (MACUT 6986)
DEM. REP. OF CONGO, Katanga, 1911, purchased from Baj- 1 Mount notti. Adult
1 Skull

\section{Genus Colobus Illiger, 1811}

DEM. REP. OF CONGO, Katanga, 1911, purchased from Baj- 1 Skull notti. Adult

Colobus guereza guereza Rüppell, 1835
MZUT T 14
(783)
(MACUT 1804)

MZUT T 47

(2730, M131)

(MACUT 7302)

MZUT T 136 (2014, M130)

MZUT T 139 (2015, M190)
ETHIOPIA, 1846, purchased from d'Hericourt 1 Mount

1 Skull

ETHIOPIA, Addis Abeba, 1916, from Bajnotti 1 Mount

[Labeled as Colubus sp.]

1 Skull

w.d., 1906, Sella don, purchased at Djibouti. Cited in Camera- 1 Mount no (1909a).

ETHIOPIA, Addis Abeba, 1907, from Bajnotti 1 Mount

Colobus guereza caudatus Thomas, 1885

MZUT T 133 (1878, M127)

MZUT T $134 \quad$ (1879, M128)

MZUT T $135 \quad$ (1880, M129)
KENYA, Mombasa, 1905, purchased from Bajnotti. Cited in 1 Mount Camerano (1909a)

KENYA, Mombasa, 1905, purchased from Bajnotti

1 Mount

KENYA, Mombasa, 1905, purchased from Bajnotti

1 Mount 
Colobus guereza occidentalis Rochebrune, 1887

MZUT T 185 (MACUT 6807)

MZUT T 275 (MACUT 6805)

MZUT T 384 (MACUT 6806)
UGANDA, Ruwenzori, Toro forest, Aug 1906, S.A.R. Duca 1 Skeleton degli Abruzzi exped. Cited by Camerano (1909a)

UGANDA, Entebbe, 1907, from Schulter. Juvenile. Cited in 1 Skull Camerano (1909a)

UGANDA, Entebbe, 1907, from Schulter. Cited in Camerano 1 Skull (1909a)

Colobus polykomos (Zimmermann, 1780)

MZUT T 385 (MACUT 6809)

LIBERIA, 1907, Buttikofer exped. Adult. Cited in Camerano (1909a)

Colobus vellerosus (I. Geoffroy Saint-Hilaire, 1834)

MZUT T 138 (243, M143)

(MACUT 28)

GHANA, 1851, from Franck. Adult. Cited in Camerano (1909a)

1 Mount

1 Skull

Colobus sp.

MZUT T $156 \quad$ (314, M135)

$$
\text { w.d., } 1878
$$

1 Mount

MZUT T 249 (MACUT 7706)

MZUT T $260 \quad$ (MACUT 7707)

DEM. REP. OF CONGO, 1916, Neri don.

1 Skull

DEM. REP. OF CONGO, Neri don.

1 Skull

DEM. REP. OF CONGO, 1916, Neri don.

1 Skull

DEM. REP. OF CONGO, 1916, Neri don.

1 Skull

w.d. Adult

1 Skull

Genus Nasalis (É. Geoffroy Saint-Hilaire, 1812)

Nasalis larvatus (van Wurmb, 1787)

MZUT T 155 (781)

(MACUT 53)

Piliocolobus badius (Kerr, 1792)

MZUT T 386

(MACUT 6810)
INDONESIA, Sumatra, Jan 1845, from Franck don.

[Locality probably wrong]

1 Skull

\section{Genus Piliocolobus Rochebrune, 1877}

LIBERIA, 1907, purchased from Schultz, ex Buttikofer exped. 1 Skeleton Cited in Camerano (1909a)

Genus Presbytis Eschscholtz, 1821

Presbytis femoralis (Martin, 1838)

MZUT T $151 \quad$ (386, M175)

INDONESIA, Borneo, 1853, Bonomi exped.

1 Mount

[Locality probably wrong]

Presbytis melalophos (Raffles, 1821)

MZUT T $150 \quad$ (834, M176)

INDONESIA, Sumatra, 1846, from Franck.

1 Mount

Genus Pygathrix (É. Geoffroy Saint-Hilaire, 1812)

Pygathrix nemaeus (Linnaeus, 1771)

MZUT T $146 \quad$ (1292, M169)

MZUT T 147 (1291, M170) w.d., 1882, Michela don. M

w.d., 1882, Michela don. M
1 Mount

1 Mount

Genus Semnopithecus Desmarest, 1822

Semnopithecus ajax (Pocock, 1928)

MZUT T 311 (MACUT 29) w.d.

1 partial Skull 
Semnopithecus entellus (Dufresne, 1797)

MZUT T $149 \quad$ (1216, M173) w.d., 1878, Polar don.

1 Mount

[Labeled as Semnopithecus sp.]

Semnopithecus vetulus (Erxleben, 1777)

MZUT T $93 \quad$ (1301, M99) w.d., 1885

1 Mount

Semnopithecus sp.

MZUT T $153 \quad(1105$, M178)

ITALY, Piemonte, Torino, 10 Mar 1874, from Torino Zoologi- 1 Mount cal Garden, dead in captivity [Labeled as Presbytis sp.]

MZUT T $371 \quad$ (MACUT 5411, w.d., 1902, Juvenile

1 Skull 5412)

1 Skeleton

Genus Trachypithecus Reichenbach, 1862

Trachypithecus cristatus (Raffles, 1821)

MZUT T 148 (821, M172)

INDONESIA, Java, Apr 1846, from Franck

1 Mount

[Locality probably wrong]

Trachypithecus obscurus (Reid, 1837)

MZUT T 152 (1711, M177)

(MACUT 5124)

Cercopithecidae indet.

MZUT T 382 (MACUT 6335)

Hylobates agilis (F. Cuvier, 1821)

$\begin{array}{ll}\text { MZUT T } 49 & (2, \text { M180) } \\ \text { MZUT T } 161 & (713)\end{array}$

Hylobates alibarbis (Lyon, 1911)

MZUT T $158 \quad$ (742, M179)

Hylobates lar (Linnaeus, 1771)

MZUT T $159 \quad$ (777)

MZUT T $160 \quad$ (778)

Hylobates moloch (Audebert, 1798)

MZUT T $50 \quad$ (1412, M182)

(MACUT 30, 3746)
MYANMAR, Thao, 10 Mar 1888, Fea exped. Adult

INDONESIA, Sumatra, 1905, Silva don.

Family HYLOBATIDAE (Gray, 1871)

Genus Hylobates Illiger, 1811

INDONESIA, Sumatra, Nov 1831, Bonomi don. Immature

1 Mount

INDONESIA, Sumatra, Ago 1845, from Franck

1 Mount

INDONESIA, Borneo, Feb 1846, from Franck

1 Mount

INDIA, Shrirangapattana, Apr 1846, from Franck

1 Mount

INDIA, Coromandel Coast, Apr 1846, from Franck

[Labeled as H. agilis]

1 Mount

ITALY, Piemonte, Torino, From Torino Zoological Garden, dead in captivity in 1886 . Adult

1 Mount

1 Skull

1 Skeleton

Genus Symphalangus (Gloger, 1841)

Symphalangus syndactylus (Raffles, 1821)

MZUT T $172 \quad$ (1, M183)

MZUT T 186

(MACUT 5343)

w.d., 1902

CATARRHINI indet.

MZUT T 191

(MACUT 4170 4710)

MZUT T 196
1 Mount

1 Skeleton

1 Skeleton

1 Skull

1 Skeleton 


\begin{tabular}{|c|c|c|c|}
\hline MZUT T 199 & (MACUT 4248) & w.d. & 1 Skeleton \\
\hline MZUT T 201 & (MACUT 5341) & w.d., 1902 & 1 Skeleton \\
\hline MZUT T 202 & (MACUT 4764) & w.d., Juvenile & 1 partial Skeleton \\
\hline MZUT T 203 & (MACUT 4565) & w.d., dead in captivity & 1 Skeleton \\
\hline MZUT T 204 & (MACUT 4247) & w.d. & 1 Skeleton \\
\hline MZUT T 206 & (MACUT 3555) & w.d. Juvenile & 1 Skeleton \\
\hline MZUT T 209 & & w.d. & 1 Skeleton \\
\hline MZUT T 212 & $\begin{array}{l}\text { (MACUT 5413, } \\
5414 \text { ) }\end{array}$ & w.d., 1902 & $\begin{array}{l}\text { 1 Skeleton } \\
1 \text { Skull }\end{array}$ \\
\hline MZUT T 218 & $\begin{array}{l}\text { (MACUT 5407, } \\
5408 \text { ) }\end{array}$ & w.d., 1902 & $\begin{array}{l}1 \text { Skeleton } \\
1 \text { Skull }\end{array}$ \\
\hline MZUT T 219 & $\begin{array}{l}\text { (MACUT 5403, } \\
5404)\end{array}$ & w.d., 1902. Immature & $\begin{array}{l}1 \text { Skeleton } \\
1 \text { Skull }\end{array}$ \\
\hline MZUT T 223 & $\begin{array}{l}\text { (MACUT 5169, } \\
5421,5422)\end{array}$ & w.d., 1902 & $\begin{array}{l}1 \text { partial Skeleton } \\
1 \text { Skull }\end{array}$ \\
\hline MZUT T 229 & $\begin{array}{l}\text { (MACUT 5377, } \\
5378 \text { ) }\end{array}$ & w.d., 1902. Immature & $\begin{array}{l}1 \text { Skeleton } \\
1 \text { Skull }\end{array}$ \\
\hline MZUT T 231 & $\begin{array}{l}\text { (MACUT 5381, } \\
5382 \text { ) }\end{array}$ & w.d., 1902. Immature & $\begin{array}{l}\text { 1 Skeleton } \\
1 \text { Skull }\end{array}$ \\
\hline MZUT T 232 & $\begin{array}{l}\text { (MACUT 5415, } \\
5416 \text { ) }\end{array}$ & w.d., 1902. Immature & $\begin{array}{l}1 \text { Skeleton } \\
1 \text { Skull }\end{array}$ \\
\hline MZUT T 243 & (23a, 1088), & w.d. & 1 Skull \\
\hline MZUT T 244 & (MACUT 5363) & w.d., 1902 & 1 Skull \\
\hline MZUT T 250 & (MACUT 4978) & ITALY, Piemonte, Torino, 1900 , dead in captivity & 1 Skull \\
\hline MZUT T 254 & (MACUT 4528) & w.d. Immature & 1 Skull \\
\hline MZUT T 255 & (MACUT 4526) & w.d. & 1 Skull \\
\hline MZUT T 256 & (MACUT 7709) & DEM. REP. OF CONGO, 1916, Neri don. & 1 Skull \\
\hline MZUT T 263 & (MACUT 4525) & w.d. & 1 Skull \\
\hline MZUT T 281 & (MACUT 3848) & w.d., 1896, from Bajnotti. Immature & 1 Skull \\
\hline MZUT T 282 & (MACUT 3556) & w.d. Immature & 1 Skull \\
\hline MZUT T 287 & $\begin{array}{l}\text { (MACUT 3554) } \\
(10087)\end{array}$ & w.d. & 1 Skull \\
\hline MZUT T 288 & (MACUT 4529) & w.d. & 1 Skull \\
\hline MZUT T 294 & (MACUT 4522) & w.d. & 1 Skull \\
\hline MZUT T 308 & & w.d. & 1 Skeleton \\
\hline MZUT T 309 & & w.d. & 1 Skeleton \\
\hline MZUT T 339 & (MACUT 3557) & w.d. & 1 Skull \\
\hline
\end{tabular}

Family HOMINIDAE Gray, 1825

Genus Gorilla (I. Geoffroy Saint-Hilaire, 1852)

Gorilla gorilla (Savage, 1847)

MZUT T 323 (MACUT 6)

w.d., 1862. Adult, M

1 Skeleton

MZUT T 347 (MACUT 3895)

CONGO, Sangha River, 1897, Gariazzo don. Adult, F

1 partial Skull

\section{Genus Pan Oken, 1816}

Pan troglodytes (Blaumenbach, 1775) 


$\begin{array}{llll}\text { MZUT T } 174 & (2816, \text { M186) } & \text { w.d., 1930, purchased, dead in captivity } & 1 \text { Mount } \\ \text { MZUT T } 176 & (15) & \text { GUINEA, 1831. Juvenile } & 1 \text { Mount } \\ & \text { (MACUT 6951) } & \text { w.d. } & 1 \text { Skeleton } \\ \text { MZUT T 177 } & & \text { GUINEA. Adult, F } & 1 \text { Mount } \\ \text { MZUT T } 178 & (10, \text { M184) } & \text { w.d. } & 1 \text { Mount } \\ \text { MZUT T 187 } & \text { (MACUT 3) } & \text { w.d. Adult } & 1 \text { Skeleton } \\ \text { MZUT T 387 } & \text { (MACUT 6952) } & \text { w.d., 1911, from Bajnotti. Juvenile } & 1 \text { Skeleton } \\ \text { MZUT T 391 } & \text { (MACUT 7034) } & \text { DEM. REP. OF CONGO, Aba, High Uelè, 1916, Neri don. } & 1 \text { Skeleton } \\ \text { MZUT T 398 } & \text { (MACUT 7699) } & \begin{array}{l}\text { Adult }\end{array}\end{array}$

\section{Genus Pongo Lacépède, 1799}

[Two different species actually recognised (Mittermeier et al., 2013). We do not attempt classification of these specimens that originated from captive individuals of unknown origin. All the specimens are labeled as Pongo pygmaeus]

Pongo sp.

MZUT T 173

MZUT T 175

MZUT T 180

MZUT T 188

MZUT T 189

MZUT T 190

MZUT T 193

MZUT T 257

MZUT T 301

MZUT T 390

(M189)

(M188)

(MACUT 4)

(MACUT 3839)

(MACUT 3853)

(MACUT 3840)

(MACUT 3851)

(MACUT 3833)

(MACUT 5)

(MACUT 7019) w.d.

w.d.

w.d. Juvenile

w.d., 1896. Juvenile

w.d., 1896. Juvenile

w.d., 1896. Juvenile

w.d., 1896. Juvenile

w.d., 1896, from Festa.

w.d., 8 Jul 1829, from Carini. Juvenile

INDONESIA, Borneo, 1910, from Bonarelli, dead in captivity. 1 Skeleton Immature

PRIMATES indet.

MZUT T 210

(MACUT 5379, 5380)

MZUT T 214

(MACUT 5387, 5388)

MZUT T 221

(MACUT 5385, 5386)

MZUT T 224

MZUT T 225

MZUT T 226

MZUT T 227

MZUT T 228

MZUT T 230

MZUT T 242

MZUT T 252

MZUT T 268 5410)

(MACUT 5391) w.d., 1902

(MACUT 5392, w.d., 1902. Juvenile 5394)

(MACUT 5393) 5398)

(MACUT 5405, 5406)

(MACUT 3561)
(MACUT 5409, w.d., 1902. Juvenile w.d., 1902

w.d., 1902. Juvenile

w.d., 1902. Adult

w.d. Juvenile

w.d.

w.d. Juvenile

w.d., 1902. Adult

w.d., 1902

w.d., 1902
1 Mount

1 Mount

1 Skeleton

1 partial Skeleton

1 partial Skeleton

1 partial Skeleton

1 Skeleton

1 partial Skeleton

1 Skeleton
1 Skull

1 Skeleton

1 Skull

1 Skeleton

1 Skull

1 Skeleton

1 Skull

1 Skeleton

1 partial Skeleton

1 Skeleton

1 Skull

1 partial Skeleton

1 Skull

1 Skeleton

1 Skull

1 Skeleton

1 Skull

6 isolated Teeth

1 Skeleton 


\begin{tabular}{|c|c|c|}
\hline MZUT T 269 & (MACUT 4524) & w.d. Juvenile \\
\hline MZUT T 274 & (MACUT 4527) & w.d. Juvenile \\
\hline MZUT T 284 & & w.d. Juvenile \\
\hline MZUT T 291 & (MACUT 4521) & w.d. Juvenile \\
\hline MZUT T 295 & (MACUT 5327) & w.d., 1902. Juvenile \\
\hline MZUT T 297 & (MACUT 3566) & w.d. Juvenile \\
\hline MZUT T 298 & (MACUT 4523) & w.d. Juvenile \\
\hline MZUT T 302 & (MACUT 3562) & w.d. Juvenile \\
\hline MZUT T 303 & (MACUT 3559) & w.d. Juvenile \\
\hline MZUT T 369 & $\begin{array}{l}\text { (MACUT 5383, } \\
5384 \text { ) }\end{array}$ & w.d., 1902 \\
\hline
\end{tabular}

1 Skull

1 partial Skull

1 Skeleton

1 Skull

1 Skull

1 Skull

1 Skull

1 Skull

1 Skull

1 Skull

1 Skeleton 